\title{
Circulating progenitor cells during exercise, muscle electro-stimulation and intermittent hypobaric hypoxia in patients with traumatic brain injury: A pilot study
}

Luisa Corral $^{\mathrm{a}, \mathrm{b}, *}$, Laura Conde ${ }^{\mathrm{a}}$, Elisabet Guillamób ${ }^{\mathrm{b}}$, Juan Blasi ${ }^{\mathrm{b}}$, Montserrat Juncadella ${ }^{\mathrm{a}}$, Casimiro Javierre $^{\mathrm{b}}$, Ginés Viscor ${ }^{\mathrm{b}}$ and Josep L. Ventura ${ }^{\mathrm{a}}$

${ }^{a}$ Intensive Care Unit and Neuropsychology Department, Bellvitge University Hospital, Barcelona, Spain

${ }^{\mathrm{b}}$ Department of Physiological Sciences II, Pathology and Experimental Therapeutics and Physiology and Immunology of University of Barcelona, Barcelona, Spain

\begin{abstract}
.
BACKGROUND: Circulating progenitor cells (CPC) treatments may have great potential for the recovery of neurons and brain function.

OBJECTIVE: To increase and maintain CPC with a program of exercise, muscle electro-stimulation (ME) and/or intermittenthypobaric-hypoxia (IHH), and also to study the possible improvement in physical or psychological functioning of participants with Traumatic Brain Injury (TBI).

METHODS: Twenty-one participants. Four groups: exercise and ME group (EEG), cycling group (CyG), IHH and ME group (HEG) and control group (CG). Psychological and physical stress tests were carried out. CPC were measured in blood several times during the protocol.

RESULTS: Psychological tests did not change. In the physical stress tests the $\mathrm{VO}_{2}$ uptake increased in the EEG and the CyG, and the maximal tolerated workload increased in the HEG. CPC levels increased in the last three weeks in EEG, but not in CyG, CG and HEG.

CONCLUSIONS: CPC levels increased in the last three weeks of the EEG program, but not in the other groups and we did not detect performed psychological test changes in any group. The detected aerobic capacity or workload improvement must be beneficial for the patients who have suffered TBI, but exercise type and the mechanisms involved are not clear.
\end{abstract}

Keywords: Traumatic brain injury, circulating progenitor cells, exercise, physiology

\section{Introduction}

Sequelae after traumatic brain injury (TBI) may be physical, mental and/or social, and may improve,

\footnotetext{
*Address for correspondence: Luisa Corral, Intensive Care Unit, Bellvitge University Hospital, Professor in the Department of Physiological Sciences II, University of Barcelona, Feixa Llarga s/n, L’Hospitalet de Llobregat 08907, Barcelona, Spain. Tel.: +34 932607511/+34 934024517; Fax: +34 932607963; E-mail: lcorral@bellvitgehospital.cat.
}

worsen or stay the same during lifetime (Whitnall et al., 2006). Patients with severe TBI have a long recovery period, improving significantly at least until one year post-TBI (Corral et al., 2007).

Stem cell treatments have great potential for the recovery of neurons and brain function. Neurogenesis in the adult brain is influenced by growth factors, neurotransmitters, hormones, pathological conditions, injuries and external stimuli. According to one theory, neurogenesis may be of peripheral origin, with 
circulating progenitor cells (CPC) differentiating into neurons (Elder et al., 2006). In 2009, Guo et al. showed an increase in CD34+ in rats' blood, brain tissue and brain angiogenesis post-TBI (Guo X et al., 2009).

Physical exercise, as well as improvements in the cardiovascular and musculoskeletal systems benefits cognitive function, increasing attention and decreasing cognitive decline and depression. These improvements occur both in healthy people (Ploughman, 2008) and in patients who have suffered TBI (Driver \& Ede, 2009). Physical exercise improves sequelae in patients who have suffered TBI through biochemical phenomena, neurotransmitters, neurogenesis and brain plasticity (Ploughman, 2008), but the mechanisms involved are not clear. As exercise can stimulate the increase in CPC (Mobius-Winkler et al., 2009), this may be one mechanism by which it achieves improvements in TBI patients.

Intermittent hypoxia facilitates the proliferation of neural stem cells in situ in the rat brain and the new divided cells in the subventricular zone and dentate gyrus react differently to hipoxia (Zhu et al., 2005; Zhu et al., 2005). Xu et al. showed a migration of neural progenitor cells (NPC) time-dependent, promoted by astrocytes induced by hypoxia, suggesting a role of astrocytes in NPC replacement therapy in the central nervous system (Xu et al., 2007; Xu et al., 2007). Our group has recently reported how intermittent hypobaric hypoxia (in a hypobaric chamber) with electrical muscle electro-stimulation is able to increase the concentration of CPC in peripheral blood in humans (Viscor et al., 2009).

The purpose of the current study was to find a stimulus able to increase and maintain CPC with a program of exercise, muscle electro-stimulation and/or intermittent-hypobaric-hypoxia (IHH), and also to study the possible improvement in physical or psychological functioning of participants with Traumatic Brain Injury (TBI).

\section{Method}

\subsection{Inclusion criteria}

Inclusion criteria were: patients who suffered severe TBI more than one year previously with physical or psychological sequelae, male sex, and age 20-60 years old. Patients with epilepsy were not included. The study was approved by the institution's Research Ethics Committee and informed consent was obtained from the patients. All patients had a past medical history of severe TBI and mean age was 35 (SD 7) years. Initial mean Glasgow Coma Scale (GCS) score was 6.8 and $37 \%$ suffered intracranial hypertension during Intensive Care Unit (ICU) stay.

One group of five patients carried out an exercise program of endurance, resistance and proprioception exercises, comprising three sessions per week at the University Exercise Physiology Unit and two sessions at home (Exercise Electro-stimulation group, EEG). However, only two completed the program, because one participant left voluntarily, another left due to alcohol and cannabis abuse and the other was operated during the study. In the sessions at the Exercise Physiology Unit muscle electro-stimulation was applied using the Compex Vitality ${ }^{\circledR}$ vascular and capillarization program with electrodes fixed in quadriceps and abdominal muscles (Bennie et al., 2002). Stimulation was applied at the maximum tolerated intensity (regulated individually by each subject) during two 20 -minute periods per session.

Another group of five patients followed a twelveweek cycling program (Cycling group, $\mathrm{CyG}$ ) three days per week. Subjects worked mainly on endurance carrying out moderate loads. Each session began with a warm-up phase, five minutes of abdominal work and cycling progressively until reaching $60 \%$ of maximum workload (adjusted every two weeks). The central phase, three 12-minute blocks of intervallic work, were applied at $60-80 \%$ with three minutes of active recuperation at $60 \%$. The sessions ended with five minutes of gentle cycling and compensatory back working and stretching.

In the IHH and muscle electro-stimulation group (HEG) six participants followed a program of IHH and electrical muscle stimulation of 3 days ( 3 hours/day) per week during 12 weeks. IHH sessions were carried out in the hypobaric chamber of Barcelona University simulating an altitude of $4.500 \mathrm{~m}$ during 2 hours and at the same time two sessions of 20 minutes of muscle electro-stimulation were applied (For two hours on alternate days, always from 3 to 5 p.m, subjects having followed their habitual diet and physical activity and with no detected illnesses or chronobiological changes). One participant was excluded because of headache. Muscle electro-stimulation was applied by means of Compex Vitality ${ }^{\circledR}$ vascular, capillarization program with electrodes fixed in quadriceps and abdominal muscles. Stimulation was applied at the maximal tolerated intensity (regulated individually by each experimental subject) during two periods of 20 minutes. Oxygen arte- 
rial saturation was measured at rest during each hypoxia exposure session by means of pulse-oxymeter.

The five participants in the control group (CG) followed a day of cognitive activities 1 day (1 hour/day) per week during 12 weeks, but one of them did not finished the program.

\subsection{Psychological and physical stress tests}

Psychological and physical stress tests were carried out before and after the program. Psychological tests evaluated features of language and work (verbal memory-RAVLT), Trail Making Test (TMT A and B), Stroop Test, working memory capacity and attention (WAIS III), information processing speed (WAIS III), orientation and verbal fluency (Barcelona test), executive functions (WAIS III and Tower of London tests) and estimated premorbid intelligence index (vocabulary, WAIS III). Reduced Paced Auditory Serial Addition Test (PASAT-G) was carried out in $\mathrm{CyG}$ which evaluate work memory components. Physical graded maximum stress tests were performed on a cycle ergometer increasing progressively the workload, in order to evaluate physical capacity and adaptation to different intensities of effort. CPC (CD34+) were measured in peripheral blood according to a previously used method (Viscor et al., 2009), at the beginning, every two weeks, and at the end of the program (blood samples were always obtained before the exercise sessions).

\subsection{Blood sampling, CD34 staining and flow cytometry assay}

All blood samples were obtained in the morning and following the same extraction methodology, as detailed below. Peripheral blood samples were collected by puncture of an antecubital vein and deposited into tubes treated with $0.34 \mathrm{M}$ di-potassium ethylenediaminetetraacetic acid anticoagulant. All samples were stored at room temperature and processed within six hours of arrival at the laboratory. Samples were incubated for cytometric absolute count with anti-human fluorescein isothiocyanate (FITC)-conjugated CD45 monoclonal antibody (BD Bioscience) and anti-human phycoerythrin (PE)-conjugated anti-CD34 (BD Bioscience) for $15 \mathrm{~min}$ at room temperature. Red blood cells were lysed with $1 \mathrm{ml}$ of quick lysis solution (CYT-QL-1, Cytognos) for $15 \mathrm{~min}$ at room temperature. Samples were incubated under dark conditions and analysed immediately. To ensure accuracy, reverse pipetting was used to dispense the volumes.
A single-platform protocol with Perfect-Count microspheres (CYT-PCM-50, Cytognos, Salamanca, Spain) was used according to manufacturer's instructions. The Perfect-Count microsphere system contains two different fluorospheres in a known proportion (A and $\mathrm{B}$ beads), thereby assuring the accuracy of the assay by verifying the proportion of both types of beads. Known volumes $(50 \mu \mathrm{L})$ of Perfect-Count microspheres were added to the same known volume $(50 \mu \mathrm{L})$ of stained blood using a lyse-no-wash technique, with the beads being counted along with cells. Cell viability was measured by staining the samples with the vital dye 7-aminoactinomycin D (7-AAD), as proposed by ISHAGE guidelines (Keeney et al., 1998). Samples were analysed on a FACSCanto II flow cytometer (BD Biosciences) with a 488-nm argon laser and DIVA 6.1.3 software (BD Bioscience). The gating strategy was in accordance with ISHAGE guidelines (Keeney et al., 1998).

\subsection{Statistical analysis}

Data are expressed as mean, median, standard deviation and interquartile range as appropriate. The continuous variables were compared using the MannWhitney $U$ test. Wilcoxon signed rank test and Friedman test were used for repeated measures. All tests were performed using SPSS v.13. Statistical significance was set at $P<0.05$.

\section{Results}

In the EEG, the results of psychological tests did not change. Aerobic capacity improved, with $\mathrm{VO}_{2}$ uptake increasing from 2.09 to $2.23 \mathrm{~L}$ and from 1.38 to $2.42 \mathrm{~L}$ in the two patients, and CPC levels in peripheral blood increasing in the last three weeks (Fig. 1).

In the $\mathrm{CyG}$ the psychological tests did not change, but PASAT-G test improved. A significant increase was found in $\mathrm{VO}_{2}$ uptake (Fig. 2), but not in CPC (Fig. 3).

In the HEG the physical stress test showed a significant difference in initial versus final load: $200 \mathrm{~W}$ (158-305) versus $218 \mathrm{~W}(169-316), p=0,043$. However, there were not changes in psychological tests and CPC. No other studied parameter showed changes in physical stress tests, psychological tests and CPC in CG. CPC CD34+ cells/ $\mu \mathrm{L}$ are shown in Fig. 4 since the beginning, during and after intervention in HEG and CG, and there were not statistical significant differences when Friedman test was applied for intra-subject repetitive measures. 


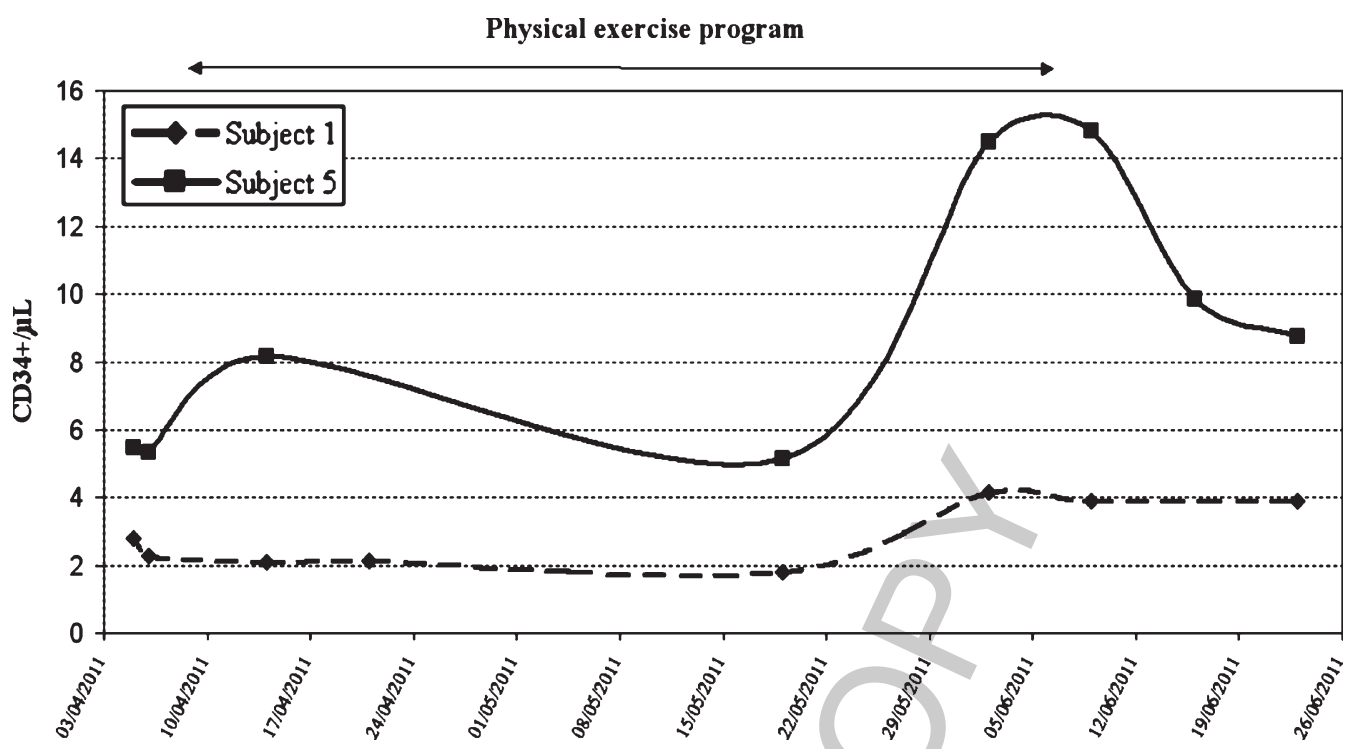

Fig. 1. CPC in the two EG subjects.

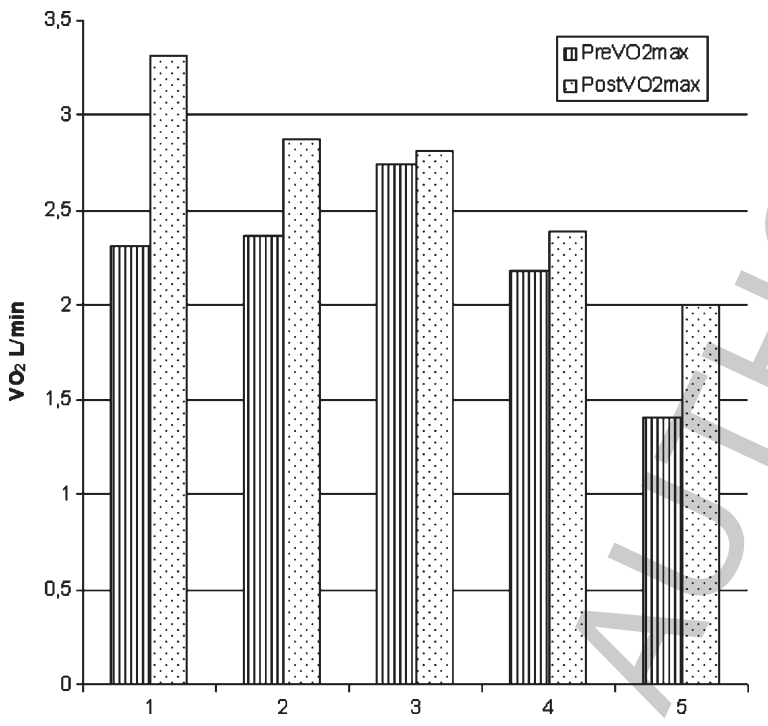

Fig. 2. Physical stress test data (CyG). PreVO2max: previous maximum oxygen uptake: $2.2+0.5 \mathrm{~L} \cdot \mathrm{min}^{-1}$ IQ range $1.8-2.3$ PostVO2max: posterior maximum oxygen uptake: $2.67+0.5$ $\mathrm{L} \cdot \mathrm{min}^{-1}$, IQ range $2.2-2.8$ (Wilcoxon test $\mathrm{p}: 0.043$ ).

\section{Discussion}

In this study, the EEG and the CyG improved their aerobic capacity and the HEG increased the workload according to physical stress test data. There were not changes in main performed psychological tests. However, only the two patients of the EEG increased CPC in the last three weeks of the program.
Exercise can improve cognitive function (Ploughman, 2008) and it may increase oxygen saturation, angiogenesis, increase neurotransmitters or upregulate neurotrophins, and brain plasticity, which mediate its beneficial effects (Ploughman, 2008). Clinical studies have shown that the reduced physical work capacity seen in TBI patients can be modified by training to increase resistance to fatigue, changes in sleeping patterns and mood and increasing self-confidence. Although not all the reported benefits of participating in physical activity have been scientifically established, physical activity in rehabilitation programs for TBI patients is important. Different types of exercise have shown benefits in recovering TBI patients, and increased social support also enhances the individual's physical self-worth, enjoyment and motivation to participate (Driver \& Ede, 2009). In this study, as expected, patients' oxygen uptake or workload increased after exercise programs, but the conducted psychological tests did not detect significant changes although patients' subjective reports reflected improved mood and well-being and PASAT-G test detected work memory improvement in CyG. Perhaps the chronic phase of the TBI made it more difficult to provoke changes, but we tried to avoid detecting changes due to the natural evolution of the illness during intervention.

Exercise seems to stimulate neurogenesis (Elder et al., 2006; Ploughman, 2008). The previous fitness level and the type, intensity and timing of the training may influence the mobilization of CPC (i.e., their 


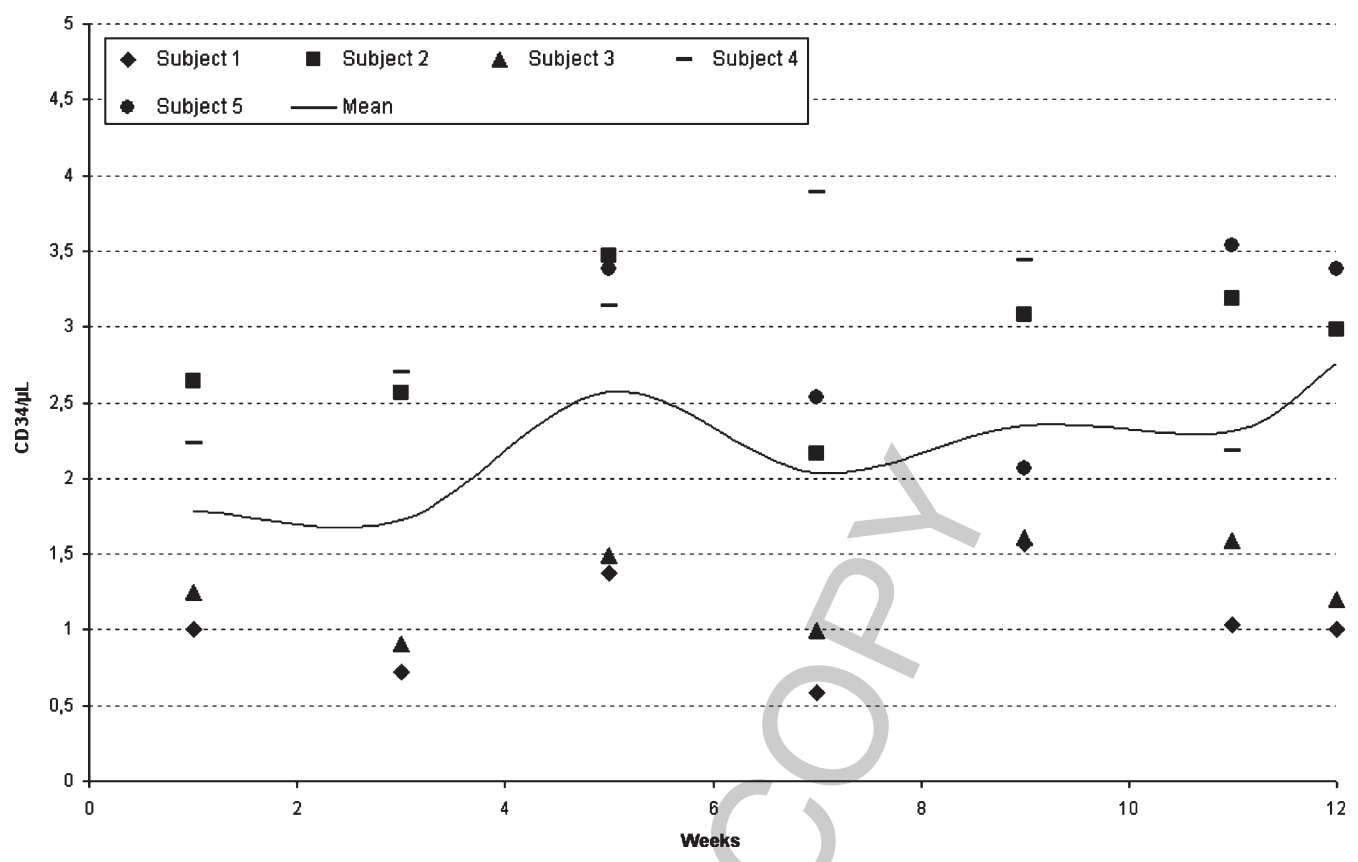

Fig. 3. CPC in the five CyG subjects.

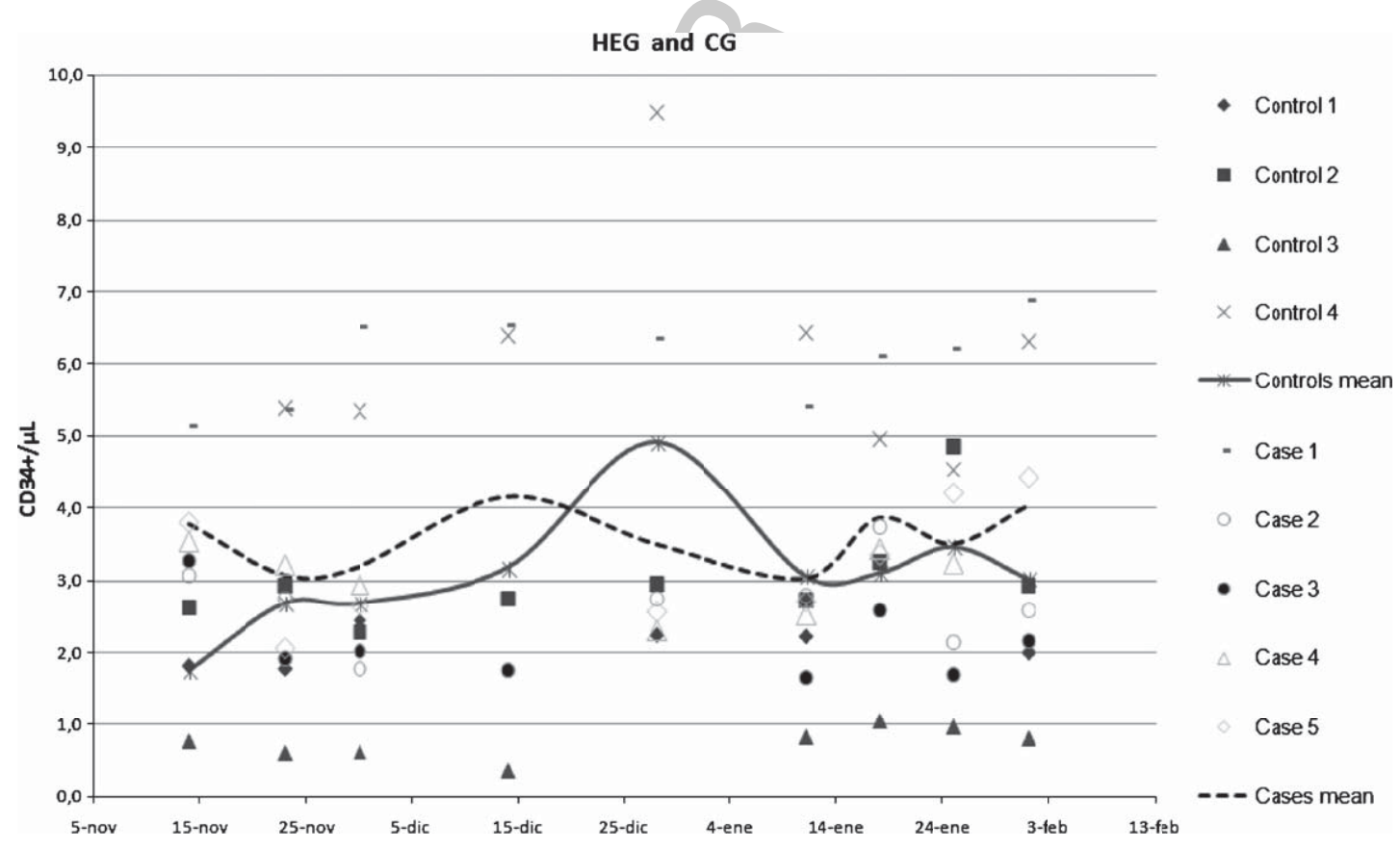

Fig. 4. CPC CD $34+$ cells $/ \mu \mathrm{L}$ in individual subjects and mean line in CG and HEG.

release into the bloodstream) and probably the tissue homing as well, thus affecting the circulating time course and emerging as a possible mechanism facilitating neurological improvement. The CPC present a time-dependent increase after exercise (Bonsignore et al., 2010; Mobius-Winkler et al., 2009) which may point to differential recruiting in peripheral tissues during prolonged endurance exercise (Bonsignore et al., 2010). Mobius-Winkler et al showed a significant timedependent increase at 210/240 min after four hours 
cycling continuously at $70 \%$ of their individual anaerobic threshold in healthy individuals (Mobius-Winkler et al., 2009). In our study, only the two EEG patients presented increase CPC. One reason may be that they had a higher workload, although we were not able to measure this in a comparative way between the groups, but we tried to apply a similar amount of trainings; another is that the two patients obtained a localized effect with muscle electro-stimulation (and probably some kind of muscle micro-injuries) while the other group performed a less focused stimulation exercise (cycling). Higher intensity of the cycling exercise (five days/week, five weeks) and previous fitness could influence CPC measurements in blood (Wang et al., 2014). CPC blood release after a stimulus also seems to follow a timedependent dynamic (Koutroumpi et al., 2012). The samples were extracted only before the sessions expecting a sustained increase in time, so that we could not detect possible post-exercise increases and oscillations. Exercise is beneficial for patients who have suffered TBI, but further studies are necessary to achieve accurate measurement of blood CPC and to determine their function and dynamics in time.

We wanted to find some stimulus to provoke CPC increased levels. The difficulty of comparing different exercise types and intensities, the possible interindividual variability in the training response and $\mathrm{CPC}$ increase, the small samples and the CPC technical variability make it difficult to identify the mechanisms involved in the different studied protocols.

Among the limitations of the study are the small sample size and the variability of the technique used to detect CPC in blood (Lysak et al., 2010). Samples were extracted only before the exercise sessions; therefore, with this experimental design it was impossible to identify fast transitory changes in CPC. Well-established psychological core measures to detect relevant TBI outcome did not detect any change, but perhaps another supplemental measure might have detected subjective changes, like PASAT-G test or others.

\section{Conclusions}

In summary, aerobic capacity or workload improvement is beneficial for patients who have suffered TBI. CPC levels increased in the last three weeks of the EEG, but not in the CyG, HEG and CG program. High intensity exercise, muscle injury and/or different timedependent mobilization may explain these increases in CPC in peripheral blood. Well-established psycho- logical core measures to detect relevant TBI outcome did not detect any change, although the PASAT-G test improved in CyG. Exercise is beneficial for patients who have suffered a TBI, but the ideal type and time and the mechanisms involved are not clear. This pilot study adds knowledge to design and continue studies to achieve accurate measurement of blood CPC and to determine their function and dynamics during different exercises in patients with TBI.

\section{Acknowledgments}

We thank the Guttman Institute for their cooperation. We would like to thank the volunteers for their participation in the present study.

\section{Declaration of interest}

The authors declare that they have no conflict of interest to disclose.

\section{Funding}

This study was supported by DEP2010-22205-C0202 grant from the Plan Nacional I+D+i 2008-2011 (Spain's Ministry of Economy and Competitiveness).

\section{References}

Bennie, S. D., Petrofsky, J. S., Nisperos, J., Tsurudome, M., \& Laymon, M. (2002). Toward the optimal waveform for electrical stimulation of human muscle. Eur J Appl Physiol, 88, 13-19.

Bonsignore, M. R., Morici, G., Riccioni, R., Huertas, A., Petrucci, E., Veca, M. et al. (2010). Hemopoietic and angiogenetic progenitors in healthy athletes: Different responses to endurance and maximal exercise. J Appl Physiol, 109, 60-67.

Corral, L., Ventura, J. L., Herrero, J. I., Monfort, J. L., Juncadella, M., Gabarros, A. et al. (2007). Improvement in GOS and GOSE scores 6 and 12 months after severe traumatic brain injury. Brain Inj, 21, 1225-1231.

Driver, S., \& Ede, A. (2009). Impact of physical activity on mood after TBI. Brain Inj, 23, 203-212.

Elder, G. A., De, G. R., \& Gama Sosa, M. A. (2006). Research update: Neurogenesis in adult brain and neuropsychiatric disorders. $M t$ Sinai J Med, 73, 931-940.

Guo, X., Liu, L., Zhang, M., Bergeron, A., Cui, Z., Dong, J., et al. (2009). Correlation of CD34 cells with tissue angiogenesis after traumatic brain injury in a rat model. Journal of Neurotrauma.

Keeney, M., Chin-Yee, I., Weir, K., Popma, J., Nayar, R., \& Sutherland, D. R. (1998). Single platform flow cytometric absolute CD34+ cell counts based on the ISHAGE guidelines. Inter- 
national Society of Hematotherapy and Graft Engineering 18. Cytometry, 34, 61-70.

Koutroumpi, M., Dimopoulos, S., Psarra, K., Kyprianou, T., \& Nanas, S. (2012). Circulating endothelial and progenitor cells: Evidence from acute and long-term exercise effects. World J Cardiol, 4, 312-326.

Lysak, D., Kalina, T., Martinek, J., Pikalova, Z., Vokurkova, D., Jaresova, M., et al. (2010). Interlaboratory variability of CD34+stem cell enumeration. A pilot study to national external quality assessment within the Czech Republic. Int J Lab Hematol, 32, e229-e236.

Mobius-Winkler, S., Hilberg, T., Menzel, K., Golla, E., Burman, A., Schuler, G., et al. (2009). Time-dependent mobilization of circulating progenitor cells during strenuous exercise in healthy individuals. J Appl Physiol, 107, 1943-1950.

Ploughman, M. (2008). Exercise is brain food: The effects of physical activity on cognitive function. Dev Neurorehabil, 11, 236-240.

Viscor, G., Javierre, C., Pages, T., Ventura, J. L., Ricart, A., MartinHenao, G., et al. (2009). Combined intermittent hypoxia and surface muscle electrostimulation as a method to increase peripheral blood progenitor cell concentration. J Transl Med, 7, 91.

Wang, J. S., Lee, M. Y., Lien, H. Y., \& Weng, T. P. (2014). Hypoxic exercise training improves cardiac/muscular hemodynamics and is associated with modulated circulating progenitor cells in sedentary men. Int J Cardiol, 170, 315-323.

Whitnall, L., McMillan, T. M., Murray, G. D., \& Teasdale, G. M. (2006). Disability in young people and adults after head injury: 57 year follow up of a prospective cohort study. J Neurol Neurosurg Psychiatry, 77, 640-645.

Xu, Q., Wang, S., Jiang, X., Zhao, Y., Gao, M., Zhang, Y., et al. (2007). Hypoxia-induced astrocytes promote the migration of neural progenitor cells via vascular endothelial factor, stem cell factor, stromal-derived factor-1alpha and monocyte chemoattractant protein-1 upregulation in vitro. Clin Exp Pharmacol Physiol, 34, 624-631.

Zhu, L. L., Zhao, T., Li, H. S., Zhao, H., Wu, L. Y., Ding, A. S., et al. (2005). Neurogenesis in the adult rat brain after intermittent hypoxia. Brain Res, 1055, 1-6. 